Classification

Physics Abstracts

81.90

\title{
Images and Modeling of the Hydromechanical Behavior of a Fracture
}

\author{
Sylvie Gentier $\left({ }^{1}\right)$, Daniel Billaux $\left({ }^{2}\right)$, Deborah Hopkins $\left({ }^{3}\right)$, Frédéric Davias $\left({ }^{1,4}\right)$ \\ and Joëlle Riss $\left({ }^{4}\right)$ \\ $\left({ }^{1}\right)$ BRGM, Direction de la Recherche, B.P. 6009, 45060 Orléans Cedex, France \\ $\left({ }^{2}\right)$ Itasca Consultants, 40 Avenue de Collongue, 69130 Ecully, France \\ $\left({ }^{3}\right)$ Lawrence Berkeley National Laboratory, 1 Cyclotron Road, Berkeley, CA 94720, USA \\ $\left({ }^{4}\right)$ Université de Bordeaux 1, Avenue des facultés, 33405 Talence Cedex, France
}

Résumé. - La compréhension du comportement hydromécanique d'une fracture est un enjeu important pour la prédiction des effets des aménagements humains dans des massifs rocheux fracturés. Lanalyse d'images est un outil puissant pour faire le lien entre la géométrie de la fracture et les différentes modélisations de son comportement hydromécanique.

\begin{abstract}
Understanding the hydromechanical behavior of fractures is a fundamental research issue because of the need to understand the effects of human engineering in fractured rock masses. The research presented in this paper is based on the use of image processing to determine the relationships between fracture geometry and hydromechanical behavior.
\end{abstract}

\section{Introduction}

Rock masses are criss-crossed by discontinuities that are called fractures. Understanding the behavior of fractures is fundamental to almost every engineering concern in rock (mining, tunneling, production of oil and natural gas, and waste management). Fractures are major conduits for fluids, and often dominate the mechanical and hydrologicalysical behavior of a rock mass.

Fractures can be envisioned as two rough surfaces in partial contact. The surface roughness, and typically sparse contact area, result in a void space between the fracture surfaces with a complex geometry. Changes in stresses in a rock mass, both natural changes and those induced by engineering activity, are reflected by changes in the void geometry in fractures as they open or close, and these changes affect the flow of fluids. The mechanical response of fractures is strongly related to the contact area, while the hydrological response is governed by the connectivity and size of the voids.

To study the relationship between void geometry and flow characteristics, a series of laboratory experiments has been performed on cylindrical rock samples containing natural fractures perpendicular to the axis of the sample. As described below, a technique has been developed 
to make casts of the void space between the fracture surfaces. Images of these casts are analyzed and transformed into data that are used as input to mechanical and hydrological models to study changes in fracture aperture and fluid flow as functions of the applied normal stress (force applied perpendicular to the plane of the fracture).

\section{Casting of the Fracture Voids and Image Acquisition}

Once a cast has been obtained [1], a pixel map is recorded using a video camera placed above the cast while it is illuminated from below by a powerful light source. Figure 1a shows the variations in thickness of a fracture cast in shades of grey. On the image, the darker grey corresponds to the thicker fracture voids and the brighter to the thinner fracture voids. Whereas this way leads to aperture estimation, it erases roughness information of the walls.

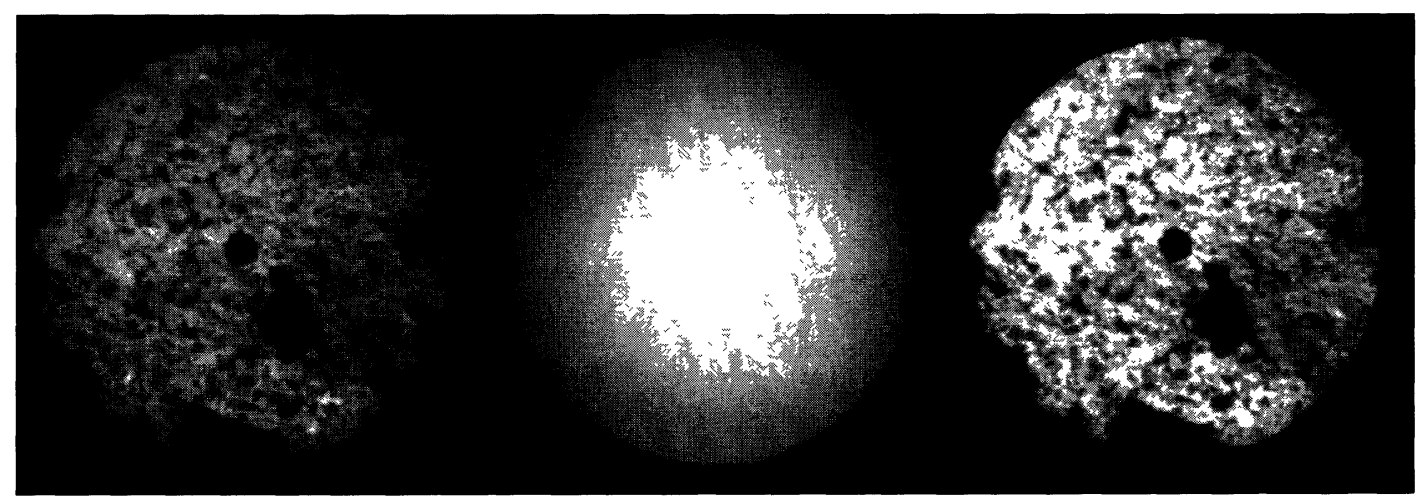

a)

b)

c)

Fig. 1. - a) Grey level image of the fracture cast (one pixel $=0.448 \mathrm{~mm} \times 0.300 \mathrm{~mm}$ ). b) Halo on the light source image. c) Cast image corrected of the light source heterogeneities.

Firstly, trends in the image due to heterogeneities of the light source must be removed. The bias is expressed on the image by a halo (Fig. 1b). The information in the grey-level image is the superposition of the light source and of the cast. To come back to the image (Fig. 1c) that we should have if the light source was perfectly uniform, the image of the emitted light powers (logarithms of the image of the light source) is substracted from the image of the light powers received (logarithms of the image of the cast). This correction assumes that the light source image and the cast image are digitized with the same location and camera settings.

Secondly, a one-to-one correspondence between grey levels and thicknesses must be defined. To do this, a "calibration wedge" is cast for each fracture cast using the same resin batch as for the "fracture void" layer. The wedge shape is obtained using a precision-engineered mold. It is digitized, and the light-source-induced trends are removed as described before. It is then easy to find a function (polynomial or exponential) establishing a correspondence between grey-levels and thicknesses. 


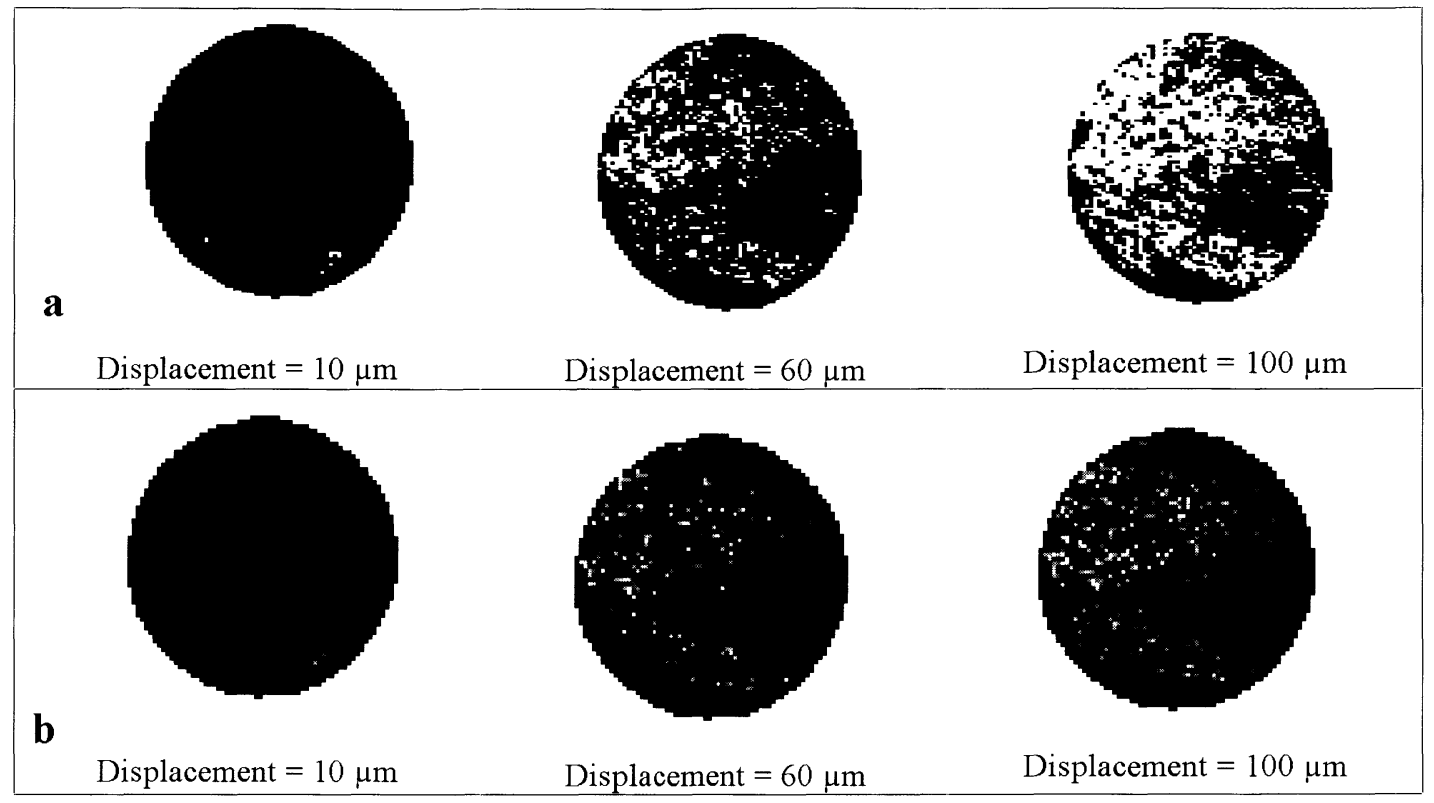

Fig. 2. - a) Evolution of the contact area with the normal stress (simplistic model). b) Evolution of the contact area with the normal stress (Hopkins' mechanical model).

\section{Mechanical Behavior of the Fracture}

The result of the preprocessing described above is a clean grey-level image paired with a thickness scale. Simple image processing includes determining the histogram of void thicknesses. The holes in the cast provide an estimate of the contact area without load (or just the weight of the sample) and the average size of the contacts. The mechanical behavior can be approached in two ways.

The first one, which is the simplest one, consists in assuming that when a normal load to the fracture is applied, the two sides of the fracture get closer uniformly on all the surface of the fracture. This phenomenon can be conveyed by successive thresholds corresponding to the progressive closure of the fracture (Fig. 2a). At each step of closure corresponding to an increasing normal load, one can easily get an estimate of the number and of the sizes of contacts. This approach leads to an overestimation of the contact area.

The second one is a real mechanical approach. A mechanical model has been developed by Hopkins [2]. The aim of this model is to predict the closure of a fracture as a function of the normal load which is applied on it. In this model, it is assumed that a joint can be modeled by two parallel half-spaces separated by variable height asperities. A normal load results in displacement across the joint due to the compression of the asperities and the deformation of the half-spaces defining the joint. The displacement of the half-spaces is calculated from the Boussinesq solution for a deformation of a semi-infinite half-space under a distributed load. The asperities are modeled as disks and their deformation is calculated from the elastic compression of the disks. The principle of superposition is used to account for mechanical interaction between the asperities; i.e. it is assumed that displacement at any point on the half-spaces is a linear combination of the displacements due to all forces acting on asperities in the region. Given the location and height of each asperity, a displacement boundary condition is specified and numerical methods are used to find the distribution of forces across the asperities that minimizes the strain energy in the system. 


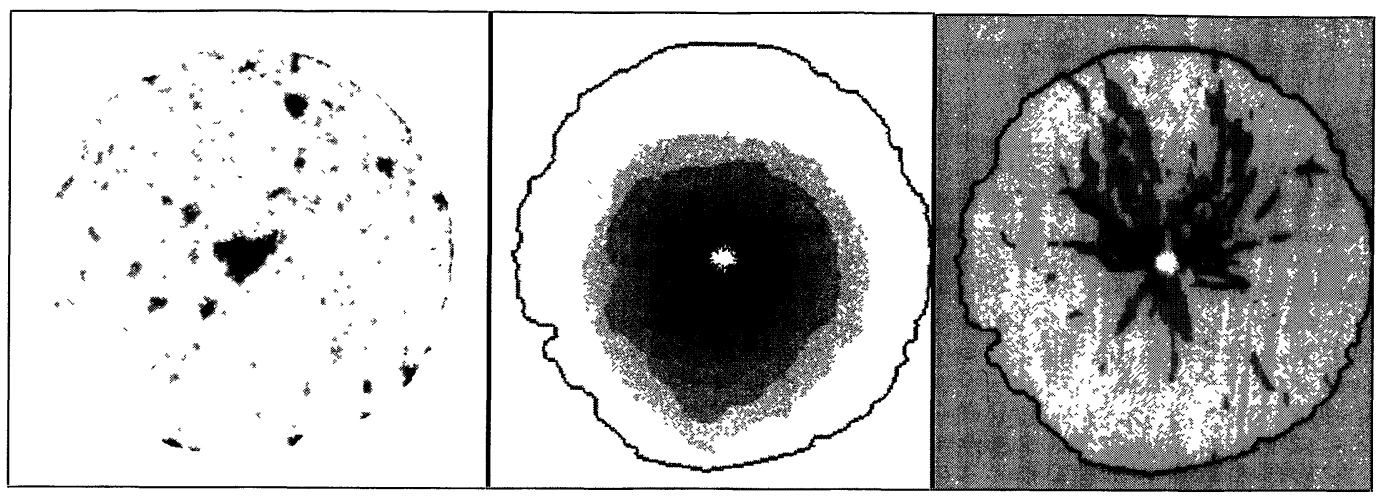

a)

b)

c)

Fig. 3. - a) Cast image. b) Map of the fluid pressure in the fracture - Darker is the grey level, higher is the pressure (3FLO Model). c) Map of the flow in the fracture - Darker is the grey level, higher is the flow rate (3FLO Model).

To be able to use this model, we need to introduce the image of the voids as basic data of the model. The grey-level image of $512 \times 512$ pixels is transformed in a regular grid. At each pixel corresponds a $(x, y)$ and at each height of void associated to each pixel corresponds a height of asperity. Each coordonate $(x, y)$ is considered as the centre of a disk. These data combined with the mechanical properties of the material are introduced in the model. In return the deformation of each asperity and the force which is applied on each asperity in contact are obtained. The result which is expressed as ( $x, y$, force) is transformed back in pixels. These new images (Fig. 2b) stemming from the mechanical calculation show the evolution of the voids when the normal load applied on the fracture increases. The grey levels on these images are proportional to the applied force.

The different kinds of images got through the two approaches permit to have a better understanding of the mechanical behavior of the fracture. The second model shows that the contact area is not as long as the first model could show. This result may have great consequences on the flow through the fracture.

\section{Hydraulic Behavior of the Fracture}

In order to study flow in the fracture, the conceptually simplest method would be to assign to each pixel of the image a transmissivity deduced from its thickness, and then to perform a numerical simulation, using finite elements or finite differences. Such a method requires powerful computers or the ability to reduce the number of points without loosing the major geometrical information.

This has already been done with the finite elements code 3FLO developed by Itasca. To the map of apertures corresponding to the grey level image is substituted a regular network of pipes whose section is calculated from the aperture at each pixel in order to conserve the fracture volume. The input data reading has been adapted to read directly an image of the fracture voids. The results of such a calculation are illustrated in Figure 3. In this approach one simply constructs a correspondence between pixels of the image and a regular grid adapted to a numerical code.

Another way has been developed [3]. Typically for natural fractures, the dimensions along the "plane" vary from a couple of centimeters to a few meters, whereas the scale of the height of the void space is around a few hundred microns. Mainly for that reason, one assumes that the fluid 


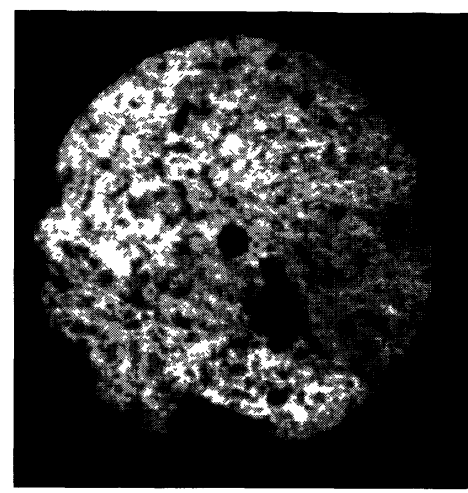

a)

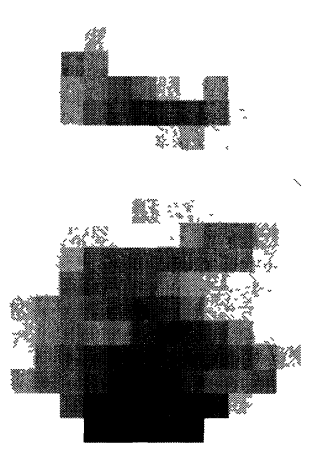

b)

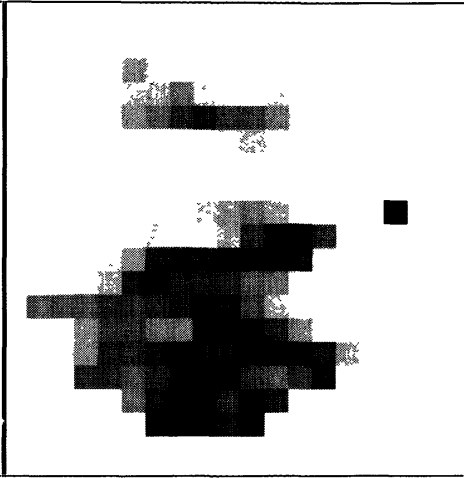

c)

Fig. 4. - a) Cast image. b) Map of the velocity in the fracture without normal stress - Darker is the grey level, lower is the velocity (Davias' Model). c) Map of the velocity in the fracture with a lower aperture due to a normal stress - Darker is the grey level, lower is the velocity (Davias' Model).Velocities have been calculated with a low number of points (around 125).

velocity variation along the height axis can be neglected. In other words, one can turn the problem naturally expressed in $3 \mathrm{D}$, into a $2 \mathrm{D}$ problem, considering mean fluid velocity vectors lying onto a projection plane which represents the fracture. Then, the void space height called aperture can be described for every point on that plane by a function called $H$. $H$ is a positive function, and the contact zones into the fracture are obviously corresponding to parts of the plane where $H$ is zero. This set of hypotheses leads to a numerical system called "shallow water" derived from the continuity equations. This system is solved by a mixed finite element method. The major difference with the classical model of flow based on the cubic law concerns the way in which the geometry of the fracture is taken into account. This model can be run conceptually with a well defined geometry, as far as this geometric information is available. The model can take into account any shape for the fracture limits, and also any number of contact zones of any shape. The aperture variation is entirely taken into account by the function $H$. All these abilities allow the model to simulate the tortuous character of the flow, experimentally observed. In this case one has to adjust the function $H$ to the void image. Figure 4 illustrates this kind of flow calculations.

Unlike the models presented above, the last way to model the flow in a fracture is based on the observed fact that, in most fractures, the flow is organized in channels and that all the more so when the normal load is high. Instead of using unnecessary details, a discretizing technique derived from a classical mathematical morphology algorithm, skeletonization [4], is therefore used. This technique accounts in a natural way for preferential flow paths. The image is first binarized using a given threshold (Fig. 5a). Flow is supposed to occur only inside a pixel with a grey-level above the threshold. The black and white image obtained is then simplified and reduced to its skeleton (Fig. 5b). This new picture represents all the connections existing in the binarized image by one pixel wide channels laying at the geometrical center of these connections. The twodimensional image is thus reduced to a mesh of one-dimensional channels. Dead-ends and small loops are then discarded (Figs. 5c, d and e). Assigning to all the channels the same transmissivity would be too great a simplification, since the skeleton "turns around contact points", without taking into account free aperture variations. In fact, an area with many isolated contact point produces a skeleton with a high density of channels winding around these contact points, whereas a well-opened area with no contact point yields only a few channels on the skeleton. This drawback 


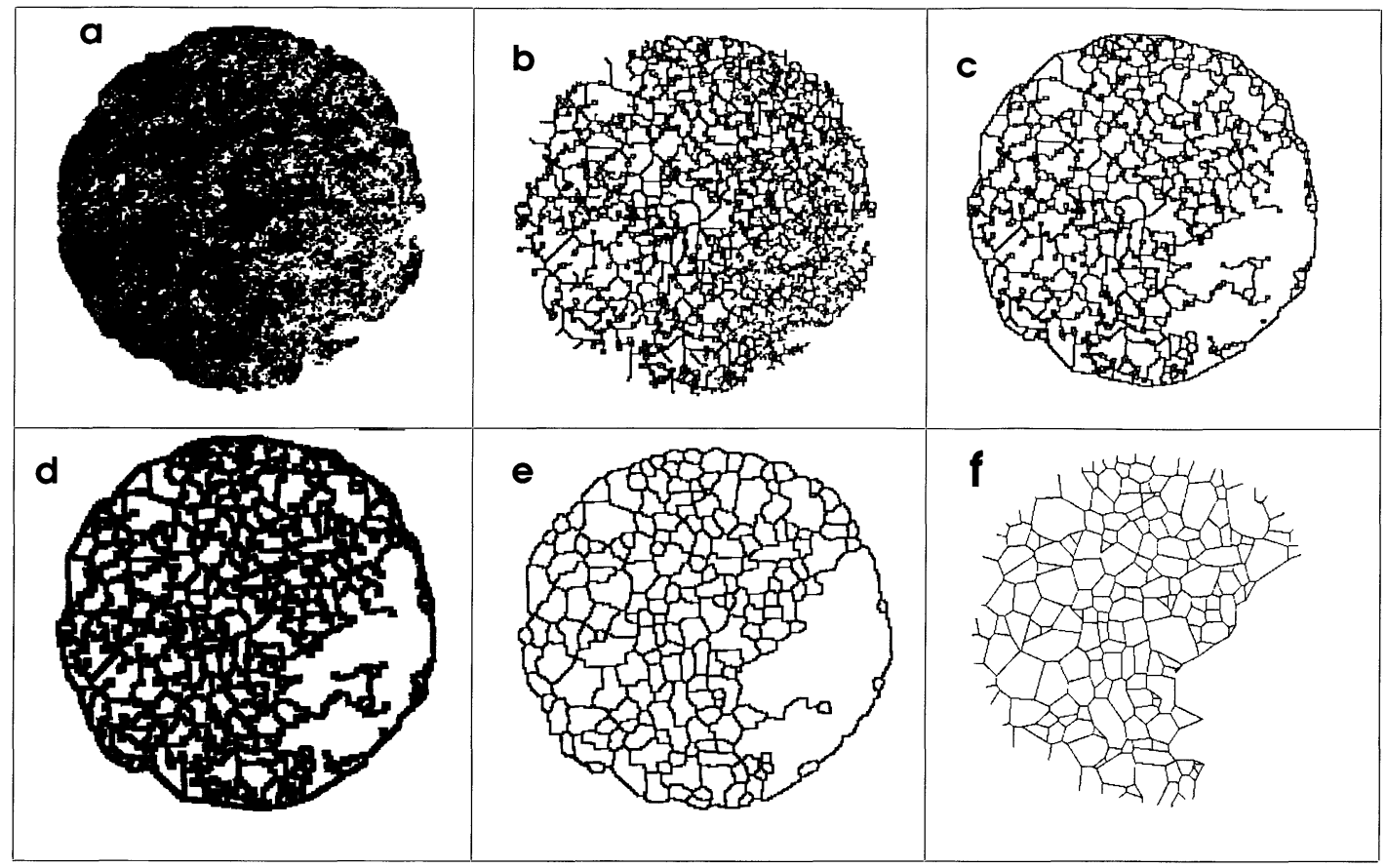

Fig. 5. - Binarized image (a). Skeleton (b). Clipped skeleton (c). Dilation (d). Skeleton (e). Mesh (f).
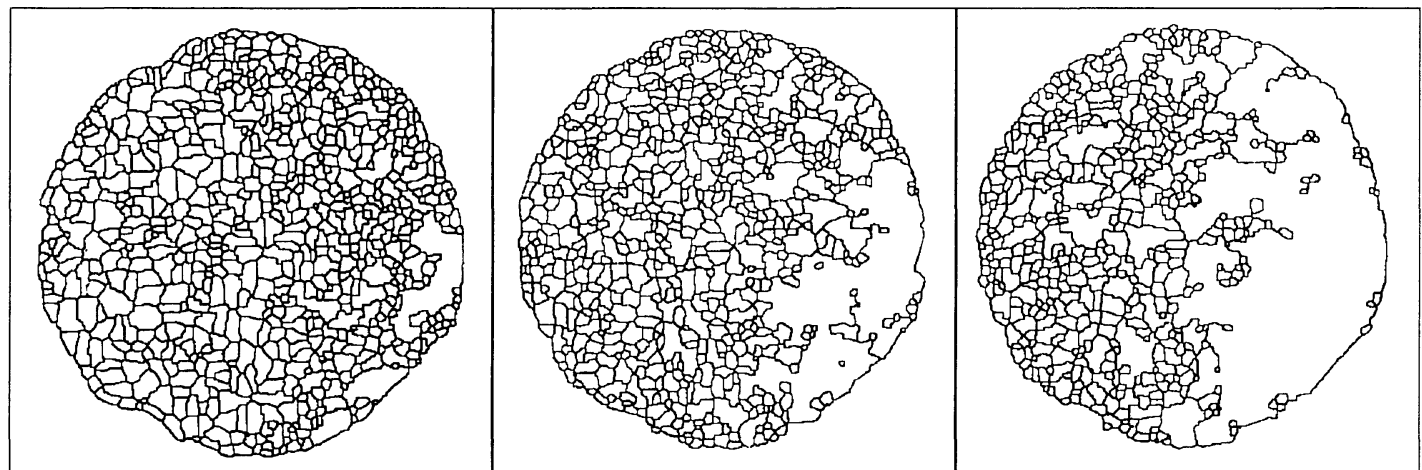

Fig. 6. - Example of evolution of a skeleton when the normal stress increases.

is suppressed by taking into account, for every channel constituting the skeleton, the importance of the flow path it represents. This is obtained by the following alteration of the skeletonization algorithm. During a given iteration, when an "on" pixel must be turned "off", its grey-level is distributed, next to it, between the pixels which stay "on" at this iteration. In this way, the integral of grey-levels upon the image stays constant at each iteration, and at the end of the skeletonization, all grey levels, and thus all thicknesses, are concentrated on the skeleton. The grey level assigned to a skeleton pixel is then the integral of the grey levels of the pixels in the flow section it represents. At this stage, a new simplified pixel map showing preferential flow paths is obtained. Figure 6 illustrates the evolution of a skeleton when the normal stress increases. In order 
to simulate the flow, one must deduce from this map a mesh constituted of one-dimensional elements, each element representing a portion of channel, bounded by two nodes representing channel intersections. This mesh is built using simple algorithms from graph theory. Firstly, a "leftmost path" algorithm [5] is used to recognize all the pixels situated on the boundary of the sample, and secondly a "breadth first search" algorithm [5] is performed to recognize all the channels, starting from the specified location of the injection hole in the centre of the cast. The length of a given element is taken equal to the number of pixels constituting it. This generally results in an overestimation of the length. The aperture, which will be used to compute the transmissivity, is given by the harmonic mean of the series of pixels the line element represents. The line mesh obtained from the skeleton in Figure 5e is shown in Figure 5f. An algorithm developed previously [6] is used to discard complex dead-ends. The flow is then computed [7] in the mesh using a classical Galerkin finite element formalism.

\section{Conclusion}

The research presented here demonstrates the important role that image processing is playing in the understanding and modeling of the hydromechanical behavior of natural fractures subjected to a normal stress. The work also demonstrates the importance of research and analysis that builds a methodology for transforming images into physically meaningful data that can be used directly, or as input to physical models.

\section{References}

[1] Gentier S., Billaux D. and Van Vliet L., Laboratory testing of the voids of a fracture, Rock Mech. Rock Eng. 22 (1989) 149-157.

[2] Hopkins D., Cook N. G.W. and Myer L.R., Normal joint stiffness as a function of spatial geometry and surface roughness, Proc. of the International Symposium on Rock Joints in Loen, Norway (Balkema, Rotterdam, 1990) pp 203-210.

[3] Davias F., Modéle d'écoulement dans une fracture dans la perspective d'une modélisation du comportement hydromécanique des milieux fracturés, to be published (1997).

[4] Coster M. and Chermant J.L., Précis d'analyse d'images (Ed. du CNRS, Paris, 1985).

[5] Baase S., Computer algorithms: introduction to designand analysis (Addison-Wesley, London, 1978).

[6] Billaux D. and Fuller P., An algorithm for mesh simplification applied to fracture hydrology, Math. Geol. 21 (1989).

[7] Billaux D. and Gentier S., Numerical and laboratory studies of flow in a fracture, Proc. of the International Symposium on Rock Joints in Loen, Norway (Balkema, Rotterdam, 1990) pp. 369-373. 